\title{
A thermodynamically general theory for convective vortices
}

\author{
By NILTON O. RENNO*, Atmospheric, Oceanic and Planetary Physics, Department of Physics, University of \\ Oxford, Oxford, OX1 3PU, UK and Department of Atmospheric, Oceanic and Space Sciences, University of Michigan, \\ Ann Arbor, MI 48109, USA
}

(Manuscript received 8 June 2007; in final form 17 March 2008)

\begin{abstract}
Convective vortices are common features of atmospheres that absorb lower-entropy-energy at higher temperatures than they reject higher-entropy-energy to space. These vortices range from small to large-scale and play an important role in the vertical transport of heat, momentum, and tracer species. Thus, the development of theoretical models for convective vortices is important to our understanding of some of the basic features of planetary atmospheres.

The heat engine framework is a useful tool for studying convective vortices. However, current theories assume that convective vortices are reversible heat engines. Since there are questions about how reversible real atmospheric heat engines are, their usefulness for studying real atmospheric vortices is somewhat controversial. In order to reduce this problem, a theory for convective vortices that includes irreversible processes is proposed.

The paper's main result is that the proposed theory provides an expression for the pressure drop along streamlines that includes the effects of irreversible processes. It is shown that a simplified version of this expression is a generalization of Bernoulli's equation to convective circulations. It is speculated that the proposed theory not only explains the intensity, but also sheds light on other basic features of convective vortices such as their physical appearance.
\end{abstract}

\section{Introduction}

Convective vortices are ubiquitous features of the atmosphere of the Earth and many other planets. They range from small-scale vortices such as dust devils, to large-scale systems such as cyclones. These vortices are hazardous and play an important role in the vertical transport of heat, momentum, and tracer species (e.g. MacPherson and Betts, 1997; Ferri et al., 2003; Renno et al., 2004). Thus, the development of theoretical models for convective vortices is important to advance our understanding of some of the basic features of planetary atmospheres.

The second law of thermodynamics states that it is impossible to achieve $100 \%$ efficiency in the conversion of heat into work (e.g. Adkins, 1968). It follows from this that a heat engine cannot operate with heat flowing from a single reservoir. Indeed, any real heat engine must absorb heat from a warmer reservoir and reject a fraction of it to a colder reservoir while doing work. In a reversible heat engine the difference between the heat input and the heat output is entirely converted into work. Energy is not wasted into irreversible process during the conversion of heat

\footnotetext{
*Correspondence.

e-mail: renno@alum.mit.edu

DOI: $10.1111 / \mathrm{j} .1600-0870.2008 .00331 . x$
}

into work. The reversible heat engine framework has been applied to hurricanes (Emanuel, 1986, 1988), Arctic lows (Emanuel and Rotunno, 1989), convective plumes (Renno and Ingersoll, 1996; Emanuel and Bister, 1996), dust devils (Renno et al., 1998, 2000), waterspouts (Renno and Bluestein, 2001), mesoscale convective circulations (Souza et al., 2000) and global circulations (Adams and Renno, 2005).

Current heat engine theories for convective vortices only calculate the pressure drop along the surface (see Renno et al., 1998). In addition, they neglect the effects of changes in kinetic energy on the pressure drop. Except for a short discussion by Renno (2001), current theories also neglect irreversible processes. Pauluis et al. (2000), Pauluis and Held (2002a, b) and Goody (2003) among others argue that, in general, atmospheric heat engines are extremely irreversible, while Emanuel (1986), Michaud (1995), Renno and Ingersoll (1996), Emanuel and Bister (1996), Renno et al. (1998, 2000), Renno and Bluestein (2001) among a few others argue that, to a first approximation, atmospheric heat engines are reversible. Since the determination of how reversible atmospheric heat engines are is essential for the determination of their intensity, it is important to include the effects of irreversible processes on theories for them.

In this paper, the heat engine framework as currently described in the literature is generalized to: (i) explicitly include the effects of irreversible processes on the intensity of convective vortices; 
(ii) calculate the pressure drop between the ambient and any point along undiluted convective updrafts, not just between the ambient and the vortex centre as done previously; (iii) include the effects of changes in air parcels' kinetic energy on the pressure drop and (iv) calculate the pressure at the interior of the vortex. It is shown, for the first time, that an expression for the pressure drop is a generalization of Bernoulli's equation to convective circulations. Finally, the proposed theory is used to calculate the maximum intensity of a variety of convective vortices and to speculate on the physical reasons for their physical appearance.

\section{Theory}

\subsection{The pressure drop}

The maximum bulk intensity of convective vortices can be calculated assuming that they reach steady state (Renno et al., 1998). In this case, the energy equation for a parcel of air follows from the dot product of the velocity vector with the equation of motion

$\mathrm{d}\left(\frac{1}{2} v^{2}+g z\right)+\alpha \mathrm{d} p=-\vec{f} \cdot \mathrm{d} \vec{l}$

where $v$ is the magnitude of the vector velocity, $g$ the gravity acceleration, $z$ the height above a reference level, $\alpha$ the specific volume, $p$ the static pressure, $\vec{f}$ the frictional force per unit mass and $\mathrm{d} \vec{l}$ an incremental distance along the air parcel trajectory.

Neglecting the heat capacity of water and assuming that the work substance is dry air, the first law of thermodynamics can be written as

$\mathrm{d} Q \approx \mathrm{d}\left(c_{v} T+l_{v} q\right)+p \mathrm{~d} \alpha+\mathrm{d} W_{\mathrm{irr}}$ or

$\mathrm{d} Q \approx \mathrm{d}\left(c_{p} T+l_{v} q\right)-\alpha \mathrm{d} p+\mathrm{d} W_{\mathrm{irr}}$,

where $\mathrm{d} Q$ is the heat input per unit mass, $c_{v}$ and $c_{p}$ are, respectively, the specific heat capacities of air at constant volume and constant pressure, $T$ the absolute temperature, $l_{v}$ the latent heat of vaporization (the difference between the latent heats at constant volume and constant pressure, and their dependence on temperature are neglected), $q$ the specific humidity and the irreversible work (per unit mass) opposing the expansions and compressions is defined as

$\mathrm{d} W_{\mathrm{irr}} \equiv \mathrm{d} Q-\mathrm{d} U-p \mathrm{~d} \alpha$,

where $\mathrm{d} U \approx \mathrm{d}\left(c_{v} T+l_{v} q\right)$ is the change in the airparcel's internal energy per unit mass. According to this definition, irreversible work includes all processes that reduce the work of expansion from the value that would result from equivalent reversible processes. This includes the work done by viscous and turbulent shear stresses opposing the expansions and compressions, and other irreversible processes caused by the fact that natural thermodynamic processes are not infinitely slow (e.g. Adkins, 1968). The heat input term includes diffusive, radiative and frictional heating as discussed in detail in section 5 of Renno and Ingersoll (1996).

Integrating eq. (1) around a convective circulation such as that illustrated in Fig. 1, we get

$\oint \alpha \mathrm{d} p \approx-\oint \vec{f} \cdot \mathrm{d} \vec{l}$

which states that in steady-state, the pressure work balances the frictional loss of energy (note that $\vec{f} \cdot \mathrm{d} \vec{l}<0$ ). As discussed by Renno and Bluestein (2001), the integration can be done along

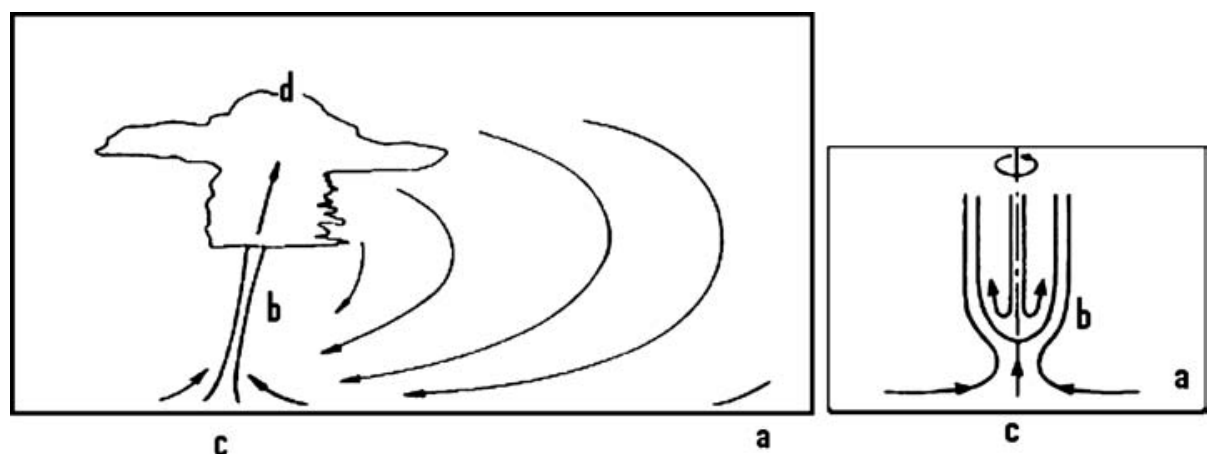

Fig. 1. (Left-hand panel) Sketch of a convective circulation with a vortex, not to scale. (Right-hand panel) Details of the circulation near the centre of a breakdown vortex. Point ' $a$ ' represents the vortex radius of influence; that is, the radius of a curve enclosing the region where flow converges towards the vortex. Point ' $c$ ' represents the near surface stagnation point at the centre of the vortex. Point 'b' represents a point along the trajectories of airparcels spiraling around the vortex in the region of maximum tangential wind. Finally, point ' $\mathrm{d}$ ' represents the top of the circulation. Contrary to previous versions, the current theory does not require that the streamlines over which the line integrals are conducted pass over point 'c.' Indeed, the current theory allows the pressure drop between any streamlines connecting point ' $a$ ' and any point ' $\mathrm{b}$ ' in the updraft to be calculated.

Scales: The horizontal extent of the convective circulation from points a to $\mathrm{c}$ ranges from about $0.1-1 \mathrm{~km}$ for dust devils, $1-100 \mathrm{~km}$ for waterspouts and tornadoes, $>1000 \mathrm{~km}$ for hurricanes. The vertical distance from points $\mathrm{c}$ to $\mathrm{d}$ ranges from about $100 \mathrm{~m}$ to a few $\mathrm{km}$ for dust devils, $1-15 \mathrm{~km}$ for tornadoes, $>10-15 \mathrm{~km}$ for hurricanes. 
a single streamline, or along a streamtube if bulk pressure drops are desired. Integrating eqs (2a) and (2b) around the circulation, we get

$\oint \mathrm{d} Q=\oint p \mathrm{~d} \alpha+\oint \mathrm{d} W_{\mathrm{irr}}=-\oint \alpha \mathrm{d} p+\oint \mathrm{d} W_{\text {irr }}$

which states that the net heat input balances the sum of the pressure work with the irreversible work. It follows from the above that

$\oint p \mathrm{~d} \alpha=-\oint \alpha \mathrm{d} p=\oint \mathrm{d} Q-\oint \mathrm{d} W_{\mathrm{irr}}$.

Integrating eq. (1) along the convective cycle from point $a$ to $b$, we get

$$
\begin{aligned}
& \int_{a}^{b} \mathrm{~d}\left(\frac{1}{2} v^{2}+g z\right)+\int_{a}^{b} \alpha \mathrm{d} p=-\int_{a}^{b} \vec{f} \cdot \mathrm{d} \vec{l} \\
& \int_{a}^{b} \alpha \mathrm{d} p=-\int_{a}^{b} \vec{f} \cdot \mathrm{d} \vec{l}-\int_{a}^{b} \mathrm{~d}\left(\frac{1}{2} v^{2}+g z\right) .
\end{aligned}
$$

Defining the thermodynamic efficiency of the circulation as

$\eta \equiv \frac{\oint p \mathrm{~d} \alpha}{\int_{a}^{c} \mathrm{~d} Q}=\frac{\oint \vec{f} \cdot \mathrm{d} \vec{l}}{\int_{a}^{c} \mathrm{~d} Q}$,

where $\int_{a}^{c} \mathrm{~d} Q$ is the heat input, and $a$ is a point at the radius of influence, defined as the farthest points from the centre of the circulation where air converges towards it, as illustrated in Fig. 1. As mentioned above, the heat input includes diffusive, radiative and frictional heating. Thus, we can write

$\oint \vec{f} \cdot \mathrm{d} \vec{l}=\eta \int_{a}^{c} \mathrm{~d} Q$.

$$
\text { Defining }
$$

$\gamma \equiv \frac{\int_{a}^{b} \vec{f} \cdot \mathrm{d} \vec{l}}{\oint \vec{f} \cdot \mathrm{d} \vec{l}}$

we get

$$
\begin{aligned}
& \int_{a}^{b} \vec{f} \cdot \mathrm{d} \vec{l}=\gamma \oint \vec{f} \cdot \mathrm{d} \vec{l} \\
& \int_{a}^{b} \vec{f} \cdot \mathrm{d} \vec{l}=\gamma \eta \int_{a}^{c} \mathrm{~d} Q .
\end{aligned}
$$

Substituting eq. (7) into (5), we get

$$
\int_{a}^{b} \alpha \mathrm{d} p=-\gamma \eta \int_{a}^{c} \mathrm{~d} Q-\int_{a}^{b} \mathrm{~d}\left(\frac{1}{2} v^{2}+g z\right) .
$$

Since, to a first approximation the convective updraft is either dry or moist adiabatic, we have that

$\int_{a}^{c} \mathrm{~d} Q=\int_{a}^{b} \mathrm{~d} Q$,

whenever point $b$ is between points $c$ and $d$ as illustrated in Fig. 1, we can write

$$
\int_{a}^{b} \alpha \mathrm{d} p=-\gamma \eta \int_{a}^{b} \mathrm{~d} Q-\int_{a}^{b} \mathrm{~d}\left(\frac{1}{2} v^{2}+g z\right) .
$$

Integrating eq. (2b) from point $a$ to $b$, we get

$$
\int_{a}^{b} \mathrm{~d} Q \approx \int_{a}^{b} \mathrm{~d}\left(c_{p} T+l_{v} q\right)-\int_{a}^{b} \alpha \mathrm{d} p+\int_{a}^{b} \mathrm{~d} W_{\mathrm{irr}} .
$$

Substituting eq. (9) into (8), we get

$$
\begin{aligned}
(1-\gamma \eta) \int_{a}^{b} \alpha \mathrm{d} p \approx & -\gamma \eta\left[\int_{a}^{b} \mathrm{~d}\left(c_{p} T+l_{v} q\right)+\int_{a}^{b} \mathrm{~d} W_{\text {irr }}\right] \\
& -\int_{a}^{b} \mathrm{~d}\left(\frac{1}{2} v^{2}+g z\right) .
\end{aligned}
$$

Using the ideal gas law, we write

$$
\begin{aligned}
(1-\gamma \eta) R \bar{T} \int_{a}^{b} \mathrm{~d} \ln p \approx & -\gamma \eta\left[\int_{a}^{b} \mathrm{~d}\left(c_{p} T+l_{v} q\right)+\int_{a}^{b} \mathrm{~d} W_{\text {irr }}\right] \\
& -\int_{a}^{b} \mathrm{~d}\left(\frac{1}{2} v^{2}+g z\right)
\end{aligned}
$$

where $R$ is the gas constant, and $\bar{T}$ the $\log$-pressure weighted mean air temperature between points $a$ and $b$. Thus, we have that

$(1-\gamma \eta) R \bar{T} \ln \frac{p_{b}}{p_{a}} \approx-\gamma \eta\left[\Delta h+W_{\mathrm{irr}}^{a b}\right]-\left[\frac{\Delta v^{2}}{2}+\Delta \phi\right]$,

where $\Delta h \approx\left(c_{p} T_{b}+l_{v} q_{b}\right)-\left(c_{p} T_{a}+l_{v} q_{a}\right) \approx c_{p} \Delta T+l_{v} \Delta q$ is the change in enthalpy between points $a$ and $b, \Delta T \equiv$ $T_{b}-T_{a}$ the change in temperature, $\Delta q=q_{b}-q_{a}$ the change in specific humidity, $\frac{\Delta v^{2}}{2} \equiv \frac{1}{2}\left(v_{b}^{2}-v_{a}^{2}\right)$ the change in kinetic energy, $\Delta \phi \equiv \Delta(g z)_{b}-\Delta(g z)_{a} \approx g \Delta z$, the change in potential energy, and $\Delta z=z_{b}-z_{a}$ the change in height. The term $W_{\text {irr }}^{a b}$ represents the irreversible work of expansion done between points $a$ and $b$. After some algebra, we get

$$
\begin{aligned}
\ln \frac{p_{b}}{p_{a}} \approx & -\frac{\gamma \eta}{(1-\gamma \eta) R \bar{T}}\left[\left(c_{p} \Delta T_{n a}+l_{v} \Delta q\right)+W_{\text {irr }}^{a b}\right] \\
& -\frac{1}{2(1-\gamma \eta) R \bar{T}} \Delta v^{2}-\frac{\Delta z}{H},
\end{aligned}
$$

where $\Delta T_{n a} \equiv \Delta T-\Delta T_{a d}=\Delta T+\frac{g}{c_{p}} \Delta z$ is the difference in temperature deviation from adiabatic between points $a$ and $b$, and $H$ is a scale height defined as

$H \equiv \frac{R \bar{T}}{g}$.

It follows from eq. (11) that

$$
\begin{aligned}
\Delta p & \equiv p_{a}-p_{b} \approx p_{a}-p_{a} \exp \left\{-\frac{\gamma \eta}{(1-\gamma \eta) R \bar{T}}\right. \\
& \left.\times\left[\left(c_{p} \Delta T_{n a}+l_{v} \Delta q\right)+W_{\mathrm{irr}}^{a b}\right]-\frac{1}{2(1-\gamma \eta) R \bar{T}} \Delta v^{2}-\frac{\Delta z}{H}\right\} .
\end{aligned}
$$

Since, even for the strongest convective vortices such as tornadoes and hurricanes $\Delta p$ is only $10-15 \%$ of the ambient pressure, the argument of the exponential is small. Expanding the 
exponential in Taylor series and neglecting high order terms, we get

$$
\begin{aligned}
\Delta p \approx & \frac{\gamma \eta p_{a}}{(1-\gamma \eta) R \bar{T}}\left[\left(c_{p} \Delta T_{n a}+l_{v} \Delta q\right)+W_{\text {irr }}^{a b}\right] \\
& +\frac{p_{a}}{2(1-\gamma \eta) R \bar{T}} \Delta v^{2}+\frac{\Delta z}{H} p_{a} .
\end{aligned}
$$

In typical convective circulations and vortices $\eta \sim 0.1$ (Emanuel, 1986; Emanuel and Bister, 1996; Renno and Ingersoll, 1996; Renno et al., 1998; Renno et al., 2000; Souza et al., 2000; Renno and Bluestein, 2001; Renno et al., 2004) and $0<\gamma<1$, thus $\gamma \eta \ll 1$ and eq. (12) can be simplified to

$$
\Delta p \approx \rho_{s} \gamma \eta\left[\left(c_{p} \Delta T_{n a}+l_{v} \Delta q\right)+W_{\mathrm{irr}}^{a b}\right]+\rho_{s} \frac{\Delta v^{2}}{2}+\frac{\Delta z}{H} p_{a},
$$

where $\rho_{s}=\frac{p_{a}}{R \bar{T}}$. Equation (13) provides insights into the physical processes causing pressure changes along the trajectory of convecting air parcels. The first two terms (between parenthesis) on the right-hand side represent the pressure drop caused by changes in enthalpy. The third term represents the pressure drop necessary to balance irreversible processes opposing the air parcels' expansion as they move from point $a$ to $b$. This term includes work by viscous and turbulent friction opposing the expansion. However, it does not include work done by viscous and turbulent shear stress opposing air parcels' motion. This is included in the pressure drop on the left hand side because, in steady state, pressure work balances frictional loss of energy as described by eq. (3). The fourth term represents the pressure drop associated with increases in airparcels' kinetic energy; it indicates that the static pressure decreases where velocity increases. We speculate that this term explains why atmospheric vortices such as dust devils, waterspouts and tornadoes are frequently hollow: Low-pressure perturbations force adiabatic expansion and flow convergence in the region of maximum tangential wind; then condensation might occur and fine dust, debris or/and sea spray are forced into the region. This is discussed in detail in Section 3.2 and illustrated by the images displayed in Figs. 2 and 3. Finally, the last term represents hydrostatic pressure

Fig. 2. (Left-hand panel) Image of a dust devil showing a hollow core. (Right-hand panel) Image of a waterspout showing a hollow core as well as the darkening of the sea surface along the trajectory of air parcels spiraling towards its radius of maximum wind.

Credits: (Left-hand panel) Photograph taken in Arizona in the Summer of 2005 by the University of Michigan. (Right-hand panel) Photograph taken on September 10, 1969 from an aircraft over the Florida Keys by Dr. Joseph Golden, NOAA.
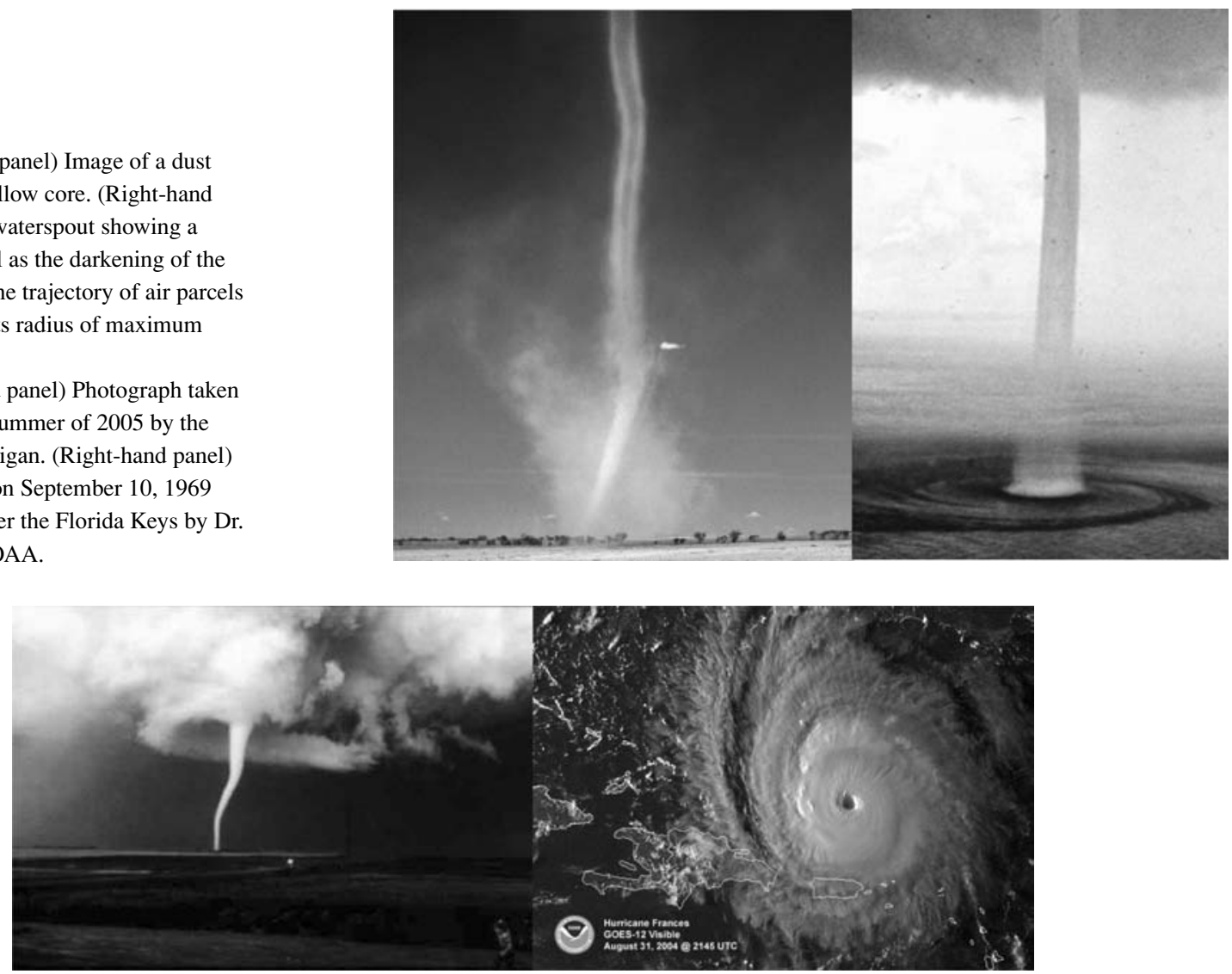

Fig. 3. Images of convective vortices showing water condensation and the formation of clouds or rainbands probably in regions of peak tangential velocities. (Left-hand panel) Image of a tornado and the associated 'wall cloud.' Wall cloud forms where the ambient air spirals toward the vortex with peak velocity. (Right-hand panel) Satellite image of a hurricane, showing spiral bands and a wall of thunderstorms surrounding the eye where the tangential velocity reaches peak values.

Credits: (Left-hand panel) Copyright by Edi Ann Otto. (Right-hand panel) National Oceanic and Atmospheric Administration. 
drops associated with changes in potential energy. It is important to note that eq. (13) is valid only along streamlines or streamtubes.

It follows from the above that the non-hydrostatic pressure drop between the radius of influence of the convective circulation at point $a$, and point $b$ in the updraft is

$$
\begin{aligned}
\Delta p_{n h} \equiv & \Delta p-\frac{\Delta z}{H} p_{a} \approx \rho_{s} \gamma \eta\left(c_{p} \Delta T_{n a}+l_{v} \Delta q\right) \\
& +\rho_{s} \frac{\Delta v^{2}}{2}+\rho_{s} \gamma \eta W_{\mathrm{irr}}^{a b} .
\end{aligned}
$$

The last term in eq. (14) is the most difficult to quantify. However, it can be calculated as a residual. This is discussed in more detail in Section 3. The parameter $\gamma$ defined by eq. (6c) is quantified next. Since atmospheric flows have large Reynolds number, making the somewhat controversial assumption (see Pauluis et al., 2000; Pauluis and Held, 2002a, b; Renno, 2001) that the frictional dissipation of energy is mostly due to turbulent viscosity on the convective scale, it can be parametrized as

$v_{\text {turb }} \sim l_{\text {edd }} \Delta v_{\text {edd }}$

where $l_{\text {edd }}$ is the eddy length scale and $\Delta v_{\text {edd }}$ the velocity across the eddies. Therefore, the magnitude of the viscous acceleration is $v_{\text {turb }} \nabla^{2} v \sim \frac{\Delta v_{\text {edd }}^{2}}{l_{\text {edd }}}$ and we have that

$\vec{f} \cdot \mathrm{d} \vec{l} \sim v_{\text {turb }}\left(\nabla^{2} v\right) \mathrm{d} l \sim \frac{\Delta v_{\text {edd }}^{2} \mathrm{~d} l}{l_{\text {edd }}}$.

Then, it follows from the definition of $\gamma$ that

$\gamma \sim \frac{\int_{a}^{b} \Delta v_{\text {edd }}^{2} \mathrm{~d} l}{\oint \Delta v_{\text {edd }}^{2} \mathrm{~d} l}$

Convective vortices have maximum velocities near the surface (Bluestein et al., 2007) and therefore the turbulent eddies generated by velocity shears are much more intense there than around the rest of the circulation. Since, energy dissipation is proportional to the square of the eddy velocity, if we take point $a$ to be at the vortex radius of influence and point $b$ anywhere between the vortex centre at point $c$ and the top of the circulation (see Fig. 1), we find $\gamma \sim 1$. The fact that frictional dissipation of energy is proportional to the square of the eddy velocity, which in turn increases dramatically near the radius of maximum wind, suggests that the bulk pressure drop associated with this term should occur near the radius of maximum wind. Non-rotating circulations or weakly rotating vortices have velocity shears that are somewhat uniform around the circulation and it follows from eq. (15b) that $\gamma \sim 0.1$. Thus, the non-hydrostatic pressure drop across the near surface branch of strongly rotating convective vortices is

$\Delta p_{n h} \approx \rho_{s} \eta\left(c_{p} \Delta T_{n a}+l_{v} \Delta q\right)+\rho_{s} \frac{\Delta v^{2}}{2}$, and across the near surface branch of weakly rotating circulations is

$\Delta p_{n h} \approx 0.1 \rho_{s} \eta\left(c_{p} \Delta T_{n a}+l_{v} \Delta q\right)+\rho_{s} \frac{\Delta v^{2}}{2}$.

\subsection{The enthalpy difference between updrafts and downdrafts}

In this subsection, we estimate the value of the change in enthalpy of airparcels moving from the low-enthalpy ambient conditions at point ' $a$ ' towards the high-enthalpy conditions at the root of the updraft at point 'c.' In steady-state, to a first approximation the enthalpy flux due to convective drafts into and out of the surface layer is balanced by surface heat flux, thus

$F_{i n} \approx M \Delta h$,

where $F_{\text {in }}$ is the surface heat flux, and

$M \approx \frac{\Delta P_{\mathrm{CBL}}}{g \tau_{R}}$,

is the convective mass flux, derived assuming that warming by subsidence is balanced by Newtonian cooling of the convective layer's atmospheric slab (see Renno and Ingersoll, 1996), $\Delta P_{\mathrm{CBL}}$ is the 'pressure thickness' of the atmospheric slab, and $\tau_{R}$ is the slab's radiative relaxation time. It follows from the above that the enthalpy change across the near surface branch convective circulation is

$\Delta h \approx\left(c_{p} \Delta T_{n a}+l_{v} \Delta q\right)-\Delta \phi \approx\left(\frac{g \tau_{R}}{\Delta P_{\mathrm{CBL}}}\right) F_{i n}$.

Equation (17) shows that, in steady-sate, the enthalpy change is directly proportional to the surface heat flux and the radiative relaxation time, and inversely proportional to the "pressure thickness' of the convective layer. Taking typical conditions in the tropics, that is $F_{\text {in }} \approx 100-200 \mathrm{~W} \mathrm{~m}^{-2}, \tau_{R} \approx 15 \mathrm{~d}$, and $\Delta P_{\mathrm{CBL}} \approx$ $800 \mathrm{hPa}$ (Renno and Ingersoll, 1996), we find $\Delta h \approx 16,000$ $32,000 \mathrm{~J} \mathrm{~kg}^{-1}$. Taking typical desert conditions, that is $F_{\text {in }} \approx$ $200-400 \mathrm{~W} \mathrm{~m}^{-2}, \tau_{R} \approx 5 \mathrm{~d}$, and $\Delta P_{\mathrm{CBL}} \approx 400 \mathrm{hPa}$ (Renno et al., 1998), we find $\Delta h \approx 20000-40000 \mathrm{~J} \mathrm{~kg}^{-1}$. Assuming flat surface $(\Delta \phi=0)$ and $\eta \approx 0.2$, it follows from eqs (16a) and (16b) that the pressure drop between the ambient and the stagnation point at the centre of the circulation $\left(\Delta v^{2}=0\right)$ are $\Delta p \approx 3-6 \mathrm{hPa}$ in typical weakly rotating tropical convective circulations, and $\Delta p \approx 32-64 \mathrm{hPa}$ in typical strongly rotating vortices. Assuming flat surface and $\eta \approx 0.1$, it follows that for typical weakly rotating desert convective circulations $\Delta p \approx 2-4 \mathrm{hPa}$ and for strongly rotating vortices $\Delta p \approx 20-40 \mathrm{hPa}$. The predictions for these two distinct regions are quite reasonable (see Renno et al., 1998; Renno and Bluestein, 2001; Samaras, 2004; Lee et al., 2004) and suggest that the proposed theory is useful. However, since the surface heat flux can vary substantially depending on local weather and surface conditions the range of extreme values can be much larger than estimated. 


\subsection{The thermodynamic efficiency}

In this subsection, the efficiency of thermodynamically general convective circulations is estimated. In eq. (6a) the thermodynamic efficiency is defined as the ratio of the work performed by the convective circulation with the heat input

$\eta \equiv \frac{\oint p \mathrm{~d} \alpha}{\int_{a}^{c} \mathrm{~d} Q}$.

The second law of thermodynamics states that for general thermodynamic processes $T \mathrm{~d} s \geq \mathrm{d} Q$, and we can write

$T \mathrm{~d} s=T \mathrm{~d} s_{\text {rev }}+T \mathrm{~d} s_{\text {irr }}=\mathrm{d} Q+T \mathrm{~d} s_{\text {irr }}$,

where $\mathrm{d} s_{\text {rev }}$ and $\mathrm{d} s_{\text {irr }}$ represent reversible and irreversible entropy changes, and $T \mathrm{~d} s_{\text {rev }} \equiv \mathrm{d} Q$ (see Renno, 2001). It follows from the above that

$\mathrm{d} Q=T \mathrm{~d} s-T \mathrm{~d} s_{\text {irr }}$.

Since $p \mathrm{~d} \alpha=\mathrm{d} Q-\mathrm{d} W_{\text {irr }}$, it follows from the above that

$\eta=\frac{\oint T \mathrm{~d} s-\oint T \mathrm{~d} s_{\mathrm{irr}}-\oint \mathrm{d} W_{\text {irr }}}{\int_{a}^{c} T \mathrm{~d} s-\int_{a}^{c} T \mathrm{~d} s_{\mathrm{irr}}}$

or

$\eta=\frac{\eta_{C}-\frac{\oint T \mathrm{~d} s_{\mathrm{irr}}}{\int_{a}^{c} T \mathrm{~d} s}-\frac{\oint \mathrm{d} W_{\mathrm{irr}}}{\int_{a}^{c} T \mathrm{~d} s}}{1-\frac{\int_{a}^{c} T \mathrm{~d} s_{\mathrm{irr}}}{\int_{a}^{c} T \mathrm{~d} s}}$,

where $\eta_{C} \equiv \frac{\oint T \mathrm{~d} s}{\int_{a}^{c} T \mathrm{~d} s}$ is the Carnot efficiency. Equation (18a) not only states that the thermodynamic efficiency of real convective heat engines approaches the Carnot efficiency only in the absence of irreversible processes, but it also quantifies the reduction in efficiency in the presence of irreversible processes. An upper bound to the thermodynamic efficiency is the Carnot value

$\eta_{\mathrm{Max}} \approx \eta_{C} \approx \frac{\Gamma_{a} Z}{2 T_{a}}$,

where $\Gamma_{a}$ is the ambient temperature lapse-rate and Zis the depth of the convective layer (see Souza et al., 2000). The factor 2 in the denominator follows from the assumption that the atmospheric slab radiates at the mean temperature of the convective layer (the temperature at $1 / 2$ the distance between the surface and the top of the convective layer).

Equation (18a) shows that irreversible processes cause smaller reduction in the thermodynamic efficiency when they occur in the heat input branch of the circulation. The main sources of irreversibility in the earth's atmosphere are associated directly or indirectly with changes of phase of water substance (Emanuel, 1994; Emanuel and Bister, 1996; Goody, 2000; Pauluis et al., 2000; Pauluis and Held, 2002a, b; Goody, 2003). Thus, nonprecipitating vortices such as dust devils and tornadoes under non-precipitating severe thunderstorms are expected to have higher thermodynamic efficiency than vortices associated with precipitating convection.

Previous studies used order of magnitude arguments (Emanuel, 1994; Emanuel and Bister, 1996), simple heuristic models (Goody, 2003), numerical models (Pauluis et al., 2000; Pauluis and Held, 2002a; Adams and Renno, 2005), and analytical calculations (Pauluis and Held, 2002b) to quantify irreversibility in moist convection. The results of these studies are not entirely consistent with each other, but in general they suggest that typical convective circulations are much more irreversible than intense convective vortices. Equations (14), (16) and (18) allow us to use real world data to quantify these irreversible processes in convective vortices. Indeed, it allows us to use measured values of the thermodynamic variables and pressure drop across convective circulations to estimate their thermodynamic efficiency, $\eta$. If the calculated value of the thermodynamic is approximately equal to the Carnot value $\eta_{\mathrm{C}}$, the convective circulation is approximately reversible. This calculation is done in Section 3.

\subsection{The vortex wall and core}

A distinctive feature of the most intense convective vortices is their funnel cloud and eye. In this subsection we study the pressure perturbations around the vortex radius of maximum tangential wind (defined as the vortex wall) and the vortex core (inwards of the vortex wall) to shed light on the flow in this region. We assume that the interior of convective vortices is mechanically forced (via turbulent eddies) by the thermodynamically forced flow spiraling from the radius of influence towards the radius of maximum tangential wind. This idea is consistent with observations that, to a first approximation, turbulent eddies force the core of most convective vortices to be in solid body rotation, while the tangential velocity outside the vortex wall can be determined from angular momentum budget of air parcels spiraling towards it (Renno and Bluestein, 2001). Indeed, our assumption is consistent with observations that to first approximation most convective vortices are Rankine vortices (e.g. Sinclair, 1966, 1969; Wurman and Gill, 2000; Renno and Bluestein, 2001; Lee et al., 2004).

Vortex flows display large structural changes when the swirl ratio, defined as the ratio of the azimuthal (or tangential) to the axial velocity (to a first approximation the vertical velocity in atmospheric vortices), is varied. Vortex breakdown are among the most important changes that may occur in the dynamics of these systems (Leibovich, 1978); it occurs when the swirl ratio exceeds a critical value. Complete vortex breakdown produces a stagnation point at the vortex axis and reversal turbulent flow in a region extending a few vortex diameters downstream of the stagnation point (Leibovich, 1978; Spohn et al. 1993, 1998). Vortex breakdown occurs in laboratory models and numerical simulations of strong tornadoes (Barcilon, 1967; Lewellen et al., 1997, 2000). Since vortex breakdown creates flow stagnation, flow reversal, and expansion of the vortex, it increases the size of the region of large pressure drop, but also decreases the magnitude of the pressure drop by reducing changes in the kinetic energy of airparcels' spiraling into the vortex as indicated by eq. (16a). 

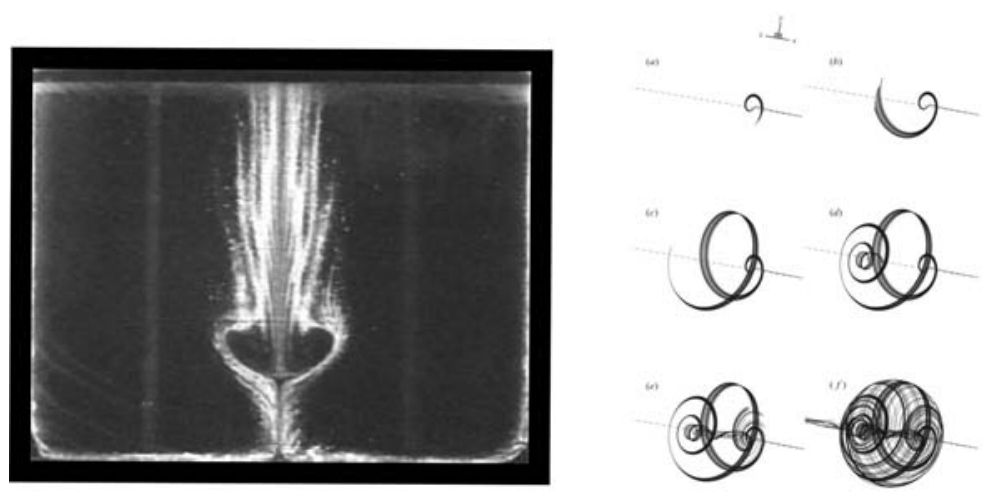

Fig. 4. (Left-hand panel) Vortex breakdown visualized by particles emitted at the boundary layer (after Spohn et al., 1998). It shows that the near surface flow converges towards the centre of the vortex. (Right-hand panel) Progression in time of trajectories of particles near the axis of rotation upstream of a breakdown vortex (After Sotiropoulos and Ventikos, 2001). It shows that the particles spiral around the breakdown bubble before entering it, them leave while spiraling around their centre.

Credits: Copyright by Cambridge University Press.
This idea is consistent with observations of pressure increases associated with vortex breakdown (Leibovich, 1978).

Laboratory simulations suggest that even in intense vortices, with breakdown bubbles, the near surface streamlines converge all the way to their centre (Spohn et al., 1993, 1998). Lagrangian trajectory analyses of numerical simulations of vortex breakdown confirm this suggestion (Sotiropoulos and Ventikos, 2001). Figure 4a reproduced from Spohn et al. (1998) indicates that the near the surface flow (or the limiting streamlines) converges towards the vortex centre, while Fig. $4 \mathrm{~b}$ reproduced from Sotiropoulos and Ventikos (2001) show that the near surface fluid rises at the centre of the vortex and spiral around the breakdown bubble before moving towards its centre, and finally leaving the bubble while spiraling around the centre of the vortex. The surface flow converges towards the centre of the vortex because friction with the surface prevents cyclostrophic balance close to the surface. In this region the vortex structure departs from the simple Rankine model. However, Wurman and Gill (2000) show that even a strong (one celled) tornado present only small departures from the Rankine model at small distances above the surface.

Assuming that to a first approximation convective vortices are Rankine vortices, their tangential velocity can be written as

$v \approx \Omega r+\delta \vartheta \quad$ for $\quad r \leq R$,

$v \approx \frac{\beta l_{a}}{r} \quad$ for $\quad r>R$,

where $\Omega=\frac{v_{R}}{R}$ is the angular velocity of the vortex wall, $v_{R}$ is the tangential velocity at the vortex wall, $R$ is the radius of the vortex wall, $r$ is the distance from the vortex centre, $\delta \vartheta$ represents small departures of the velocity from solid body rotation, $(1-\beta)$ is the fractional loss of angular momentum of airparcels spiraling toward the vortex, and $l_{a}$ the angular momentum at the vortex radius of influence (point a). Since, to a first approximation the flow in the vortex core and wall is in cyclostrophic balance we have that

$\frac{1}{\rho} \frac{\partial p}{\partial r} \approx \frac{v^{2}}{r}+\delta C$ where $\delta C$ represents departures of the acceleration from cyclostrophic balance.

Neglecting products of perturbations we find that

$\frac{1}{\rho} \frac{\partial p}{\partial r} \approx \Omega^{2} r+2 \Omega \delta \vartheta+\delta C$ for $r \leq R$,

$\frac{1}{\rho} \frac{\partial p}{\partial r} \approx \frac{\beta^{2} l_{a}^{2}}{r^{3}}+\delta C$ for $r>R$

Equtions (20a) and (20b) indicate that the pressure gradient is maximum around $R$, the radius of maximum tangential wind. Integrating eq. (20a) from the vortex radius of maximum wind, $R$ to a point $r$ in its core, we get

$\int_{p_{R}}^{p_{r}} \frac{\mathrm{d} p}{\rho} \approx \int_{R}^{r} \Omega^{2} r \mathrm{~d} r+\int_{R}^{r} 2 \Omega \delta \vartheta \mathrm{d} r+\int_{R}^{r} \delta C \mathrm{~d} r$

$\frac{p_{r}-p_{R}}{\rho} \approx \frac{\Omega^{2}}{2}\left(r^{2}-R^{2}\right)+2 \Omega \int_{R}^{r} \delta \vartheta \mathrm{d} r+\int_{R}^{r} \delta C \mathrm{~d} r$.

Now, we assume that the interior of convective vortices is mechanically forced (via turbulent eddies) by the thermodynamically forced flow spiraling from the radius of influence towards the radius of maximum tangential wind and use three steps to calculate the pressure at the vortex interior: (i) eq. (16a) is integrated from point $a$ at the ambient to a point $b$ at the radius of maximum wind, giving the pressure drop between these two points; (ii) eq. (21) is used to calculated the pressure drop between point $b$ at the radius of maximum wind and points at radius $r$ in the vortex interior and (iii) the results of the previous two steps are added to each other resulting in an expression for the pressure drop at the vortex interior as function of various thermodynamic variables. These calculations are done next. We start by re-writing eq. (16a) as

$\left(1-\frac{\Delta \mathrm{z}}{H}\right) \frac{p_{a}}{\rho_{s}}-\frac{p_{b}}{\rho_{s}} \approx \eta\left(c_{p} \Delta T_{n a}+l_{v} \Delta q\right)+\frac{\Delta v^{2}}{2}$. 
Taking point $b$ to be at the radius of maximum wind $(R)$, we write

$\left(1-\frac{\Delta \mathrm{z}}{H}\right) \frac{p_{a}}{\rho_{s}}-\frac{p_{R}}{\rho_{s}} \approx \eta\left(c_{p} \Delta T_{n a}+l_{v} \Delta q\right)+\frac{\Omega^{2} R^{2}}{2}$,

where we assume that the vortex moves with the ambient wind and therefore $\Delta v \approx \Omega R$. Using the above equation to substitute for $p_{R}$ in eq. (21), we find an expression for the non-hydrostatic pressure drop between the radius of influence (point $a$, where the velocity is zero in a frame of reference moving with the vortex) and a point in the vortex core at distances $r \leq R$ from its axis of rotation

$$
\begin{aligned}
\left(1-\frac{\Delta z}{H}\right) \frac{p_{a}}{\rho_{s}}-\frac{p_{r}}{\rho} \approx & \eta\left(c_{p} \Delta T_{n a}+l_{v} \Delta q\right) \\
& +\left(\Omega^{2} R^{2}-\frac{\Omega^{2} r^{2}}{2}\right)+2 \Omega \int_{R}^{r} \delta \vartheta \mathrm{d} r \\
& +\int_{R}^{r} \delta C \mathrm{~d} r \text { for } r \leq R,
\end{aligned}
$$

where the left-hand side terms represents the non-hydrostatic pressure drop (normalized by the air density) between the vortex's radius of influence (point $a$ ) and a point in the interior of the vortex (a point at $r \leq R$ ) at height $\Delta z$ above point $a$. The first term on the right-hand side represents the thermodynamic pressure drop (normalized by the air density), the second term represents the pressure drop due to the centrifugal acceleration and changes in kinetic energy between points in the vortex core and its radius of influence, the third term represents pressure perturbations due to departures from solid body rotation, and the last term represents pressure perturbations associated with departure from cyclostrophic balance.

Equation (22) is also a reasonable approximation just outside the region of maximum wind, that is at $r=R+\delta r$, where $\delta r$ represents a small distance relative to $R$. In this region, the pressure gradient acceleration is larger than the centrifugal acceleration because airparcels spiral towards $R$, and therefore $\delta C$ $<0$. Moreover, in these regions the reduction of air parcels angular momentum due to surface friction implies that $\delta \vartheta<0$. Thus, the last two terms of eq. (22) are $<0$ and produce increases in the pressure where the flow converges towards the vortex. This is what happens in the surface layer where friction is large and airparcels spiral all the way towards the centre of the vortex.

In the region where the vortex core is in nearly cyclostropic balance and solid body rotation (that is, where $r \leq R$ ), the last two terms of eq. (22) can be neglected and it simplifies to

$$
\frac{\Delta p_{n h}(r)}{\rho_{s}} \approx \eta\left(c_{p} \Delta T_{n a}+l_{v} \Delta q\right)+\Omega^{2} R^{2}-\frac{\Omega^{2} r^{2}}{2} \text { for } r \leq R,
$$

in a frame of reference moving with velocity $v_{a}$, where the nonhydrostatic pressure drop at the vortex interior is defined as

$\Delta p_{n h}(r) \equiv\left(1-\frac{\Delta z}{H}\right) \frac{p_{a}}{\rho_{s}}-\frac{p_{r}}{\rho}$.
Thus, where the vortex core is in nearly cyclostropic balance the pressure gradient is largest around the vortex wall $(r \approx R)$. We hypothesize that this is what happens above the surface layer where spiraling airparcels do not enter the vortex core. In addition, before steady state is reached, the maximum pressure drop might be at the vortex wall.

\section{Discussion}

In this section, we use the theory developed in Section 2 to study convective vortices ranging in size and intensity from dust devils to hurricanes. We suggest that it sheds light into the physics of these systems and predicts the formation of some of their most distinctive features such as those illustrated in Figs. 2 and 3.

The formation of convective vortices requires the presence of convection and sources of vorticity. Strong convective vortices such as dust devils, waterspouts, and tornadoes form in the presence of intense convective circulations. Renno and Bluestein (2001) derived an expression for the radius of maximum wind of convective vortices as a function of the ambient vorticity. Their results suggest that convective vortices form when there is enough vorticity present in the environment for vortices large enough to balance the destruction of velocity gradients by eddy viscosity to form. The formation of convective circulations in turn requires only the onset of convection. When only vertical temperature gradients are present, the Rayleigh number must exceed a critical value for convection to form, while convective circulations always occur in the presence of horizontal temperature gradients (Koschmieder, 1993). Circulations such as sea and lake breezes require only the presence of horizontal temperature gradients to form, but their intensity depends on the depth of the convective layer via the thermodynamic efficiency (Souza et al., 2000).

At the surface layer where torques by friction with the ground reduces the angular momentum of airparcels spiraling into the vortex centre $(\beta \sim 0)$ and airparcels reach the stagnation point at the centre of the vortex. It follows from eq. (16a) that the maximum pressure drop at the surface is

$\frac{\Delta p_{n h}^{\mathrm{SFC}}}{\rho_{s}} \approx \eta\left(c_{p} \Delta T_{n a}+l_{v} \Delta q\right)$.

Equation (24) suggests that at the surface, the maximum pressure drop occurs at the centre of the vortex. This is consistent with pressure measurements in a strong tornado (Lee et al., 2004; Samaras, 2004).

It follows from eq. (23) that just above the surface layer, where spiraling airparcels do not penetrate the interior of the vortex, the maximum pressure drop is

$\frac{\Delta p_{n h}^{\mathrm{ASL}}}{\rho_{s}} \approx \eta\left(c_{p} \Delta T_{n a}+l_{v} \Delta q\right)+\Omega^{2} R^{2}$,

where it was assumed that the heat input at both the surface and just above the surface layer are similar because the integral 
is along the limiting streamlines or streamtube (see Fig. 4). This assumption produces an upper bound to the pressure drop. Equations (23), (24) and (25) suggest that the maximum potential pressure drop is at the centre of the vortex, and just above the surface layer. The idea that the maximum pressure drop occurs just above the surface is illustrated by the results of numerical simulations (e.g. Michaels and Rafkin, 2004).

Measurements of pressure in convective vortices might not clearly show a correlation between pressure drop and kinetic energy for two reasons: (i) Only the total or stagnation pressure is usually measured, and not the static pressure. This occurs because measurement of the static pressure requires the careful placement of a probe aligned with the flow, so that the pressure ports are perpendicular to it. (ii) The pressure is usually measured only near the surface where the tangential wind speed is substantially smaller than above the surface. In Section 3.2, we speculated on the implications of the annulus of maximum pressure gradients and therefore flow convergence at the radius of maximum wind.

\subsection{Calculations of the thermodynamic efficiencies and the maximum pressure drops}

In this section we use a combination of data and theory to calculate the maximum potential pressure drop in convective vortices of various scales and intensity. Our approach to the calculations is the following: (i) eq. (18b) is used to calculate the maximum thermodynamic efficiency of the convective vortices, $\eta_{\text {Max }} \approx \eta_{C}$, taking observed values of the depth of the convective layer $(Z)$, ambient temperature lapse-rate $\left(\Gamma_{a}\right)$, and near surface ambient air temperature $\left(T_{a}\right)$; (ii) eq. (24) is used to calculate the pressure drop at the surface, $\Delta p_{n h}^{\mathrm{SFC}}$, taking $\eta \sim \eta_{\text {Max }}$ and observed values of the temperature and specific humidity perturbations, $\Delta T \sim \Delta T_{n a}$ (over flat surfaces) and $\Delta q$ and (iii) eq. (25) is used to calculate the maximum pressure perturbation, $\Delta p_{n h}^{\mathrm{ASL}}$, assuming that the maximum tangential velocity follows from a balance between the thermodynamically forced pressure gradient and centrifugal accelerations, that is that the tangential velocity at the radius of maximum wind is $v \sim \Omega R \sim \sqrt{\Delta p^{\mathrm{SFC}} / \rho}$. In this approach, agreements between calculated and measured pressure drops would suggest that $\eta \sim \eta_{C}$ and $W_{\text {irr }}^{a b} \sim 0$. Our calculations are detailed below and its main results are summarized in Table 1.

Measurements in a strong dust devil over a flat surface near Tucson, Arizona, gives $\Delta T_{n a} \approx \Delta T \approx 5 \mathrm{~K}, \Delta q \approx 0$, and $\Delta p_{n h} \approx 2-3 \mathrm{hPa}$ (Sinclair, 1966, 1969). The surface pressure at the site is $p_{a} \approx 925 \mathrm{hPa}$ and the depth of the convective layer is $Z \approx 3 \mathrm{~km}$ (Sinclair, 1966; Renno et al., 1998). It follows from eq. (18b) that $\eta_{\mathrm{Max}} \approx \eta_{C} \approx 0.05$, from eq. (24) that $\Delta p_{n h}^{\mathrm{SFC}} \approx$ $2.5 \mathrm{hPa}$, and from eq. (25) that $\Delta p_{n h}^{\mathrm{ASL}} \approx 3.5 \mathrm{hPa}$. The fact that the near surface pressure drop is of the order of the observed value suggests that $\eta \sim \eta_{C}$ and $W_{\text {irr }}^{a b} \sim 0$ in strong dust dev-
Table 1. Non-hydrostatic pressure drops between point $a$ at the ambient and at the near-surface stagnation point $c$ close to the centre of the vortex, and at the region of maximum tangential wind, just above the surface. The thermodynamic efficiency is calculated using eq. (18) and the pressure drops using eqs (24) and (25), with $c_{p}=1005 \mathrm{~J}$ $(\mathrm{kg} \mathrm{K})^{-1}$ and $l_{v}=2.05 \times 10^{6} \mathrm{~J} \mathrm{~kg}^{-1}$. Observed values of the near surface pressure drop are placed after the backslash

\begin{tabular}{lccccc}
\hline & $\gamma$ & $\eta$ & $\Delta T_{n a}(\mathrm{~K})$ & $\Delta q\left(\mathrm{~g} \mathrm{~kg}^{-1}\right)$ & $\Delta p_{n h}(\mathrm{hPa})$ \\
\hline Dust devils & 1.0 & 0.05 & 5.0 & 0 & $2-5 / 3$ \\
Weak waterspouts & 1.0 & 0.1 & 1.0 & 0.002 & $3-6 / 6$ \\
Strong waterspouts & 1.0 & 0.2 & 4.0 & 0.005 & $30-60 / 65$ \\
Tornadoes & 1.0 & 0.2 & 6.0 & 0.01 & $50-100 / 100$ \\
Hurricanes & 1.0 & 0.2 & 10 & 0.01 & $60-120 / 90$ \\
\hline
\end{tabular}

ils. Moreover, Sinclair data, reproduced in fig. 2 of Renno et al.

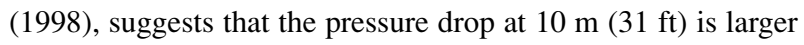

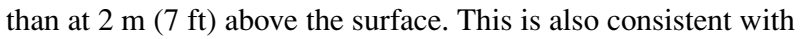
the predictions of the proposed theory.

Waterspouts usually form over tropical oceans where the surface temperature is at least about $300 \mathrm{~K}$. To a first approximation waterspouts have saturated updrafts and downdrafts, and their low-level tangential velocity can reach peak values of about $80 \mathrm{~m} \mathrm{~s}^{-1}$ (Golden, 1974; Leverson et al., 1977; Renno and Bluestein, 2001). Weak waterspouts, just strong enough to produce sea spray have maximum tangential wind speeds of about $25 \mathrm{~m} \mathrm{~s}^{-1}$. These weak waterspouts form under convective clouds at least 3-4 km deep, and have temperature and pressure perturbations of $\sim 1-2 \mathrm{~K}$ and $\sim 5-10 \mathrm{hPa}$ (Golden, 1973, 1974; Renno and Bluestein, 2001); while strong waterspouts form under convective clouds at least $\sim 10-15 \mathrm{~km}$ deep and have temperature and pressure perturbations of $\sim 2-4 \mathrm{~K}$ (Golden, 1974; Renno and Bluestein, 2001). Assuming that strong waterspouts are in cyclostrophic balance, tangential wind speeds of $80 \mathrm{~m} \mathrm{~s}^{-1}$ implies that they have pressure drops of about $65 \mathrm{hPa}$. Assuming that the updrafts and downdrafts are saturated, weak and strong waterspouts have specific humidity perturbations $\Delta q$ of about 0.002 and $0.005 \mathrm{~kg} \mathrm{~kg}^{-1}$. Assuming that ambient temperature lapse-rate is approximately moist adiabatic $\Gamma_{a} \sim 7 \mathrm{~K} \mathrm{~km}^{-1}$, it follows from eq. (18b) that $\eta_{\mathrm{Max}} \approx \eta_{C} \approx 0.05$ and 0.2 for weak and strong waterspouts, respectively. It follows from these observations and eqs (24) and (25) that $\Delta p_{n h}^{\mathrm{SFC}} \approx 3 \mathrm{hPa}$ and $\Delta p_{n h}^{\mathrm{ASL}} \approx 6 \mathrm{hPa}$ for weak waterspouts, and 30 and $60 \mathrm{hPa}$ for strong waterspouts. Since the near surface pressure drops are of the order of the observed values we conclude that $\eta \sim \eta_{C}$ and $W_{\text {irr }}^{a b} \sim 0$ in waterspouts.

The temperature and specific humidity perturbations in strong tornadoes are calculated assuming that their updrafts and downdrafts are saturated and follow the warmest (originating at the surface) and coldest (originating at the mid troposphere) adiabats intercepting atmospheric soundings taken near the tornado. The depth of the parent thunderstorm is estimated using 
the sounding's level of neutral buoyancy. An F-4 tornado that passed through Manchester, South Dakota, on June 24, 2003 is chosen for our calculation because high-quality in situ measurements were made in its funnel cloud (Lee et al., 2004). These measurements include the pressure drop at the centre of the funnel cloud. The pressure drop in this tornado was about $100 \mathrm{hPa}$, and its maximum near surface tangential wind speed was about $90 \mathrm{~m} \mathrm{~s}^{-1}$ (Lee et al., 2004). Moreover, the in situ data suggests to a first approximation this tornado is a Rankine vortex. It follows from the assumptions described above and the atmospheric sounding displayed in fig. 5 of Lee et al. (2004) that $Z \sim$ $15 \mathrm{~km}, \eta_{\text {Max }} \approx \eta_{C} \approx 0.2, \Delta T_{n a} \approx \Delta T \approx 6 \mathrm{~K}$, and $\Delta q \approx 0.01$ for this tornado. Substituting these values in eqs (24) and (25), we get $\Delta p_{n h}^{\mathrm{SFC}} \approx 50 \mathrm{hPa}$ and $\Delta p_{n h}^{\mathrm{ASL}} \approx 100 \mathrm{hPa}$. Since the near surface pressure drops are of the order of the observed values we conclude that $\eta \sim \eta_{C}$ and $W_{\text {irr }}^{\text {ab }} \sim 0$ in strong tornadoes.

We use measurements done during the landfall of hurricane Andrew to estimate the intensity of a strong hurricane. At landfall, hurricane Andrew had pressure drop of about $90 \mathrm{hPa}$ and maximum tangential wind speed of about $70 \mathrm{~m} \mathrm{~s}^{-1}$ (Landsea et al., 2004), and the sea surface temperature in the area was about 303 K. Moreover, Liu et al. (1999) argue that Andrew's eyewall convection had tops at around $15 \mathrm{~km}$, that its near surface temperature perturbation was $\sim 10 \mathrm{~K}$, and that the relative humidity increased from about $85 \%$ at the environment to $100 \%$ in the eyewall. It follows from this that and $\eta_{\text {Max }} \approx \eta_{C} \approx 0.2$, $\Delta T_{n a} \approx 10 \mathrm{~K}$, and $\Delta q \approx 0.01$. Substituting these values in eqs (24) and (25), we get $\Delta p_{n h}^{\mathrm{SFC}} \approx 60 \mathrm{hPa}$ and $\Delta p_{n h}^{\mathrm{ASL}} \approx$ $120 \mathrm{hPa}$. Since the near surface pressure drops are of the order of the observed values we conclude that $\eta \sim \eta_{C}$ and $W_{\text {irr }}^{a b} \sim$ 0 in strong hurricanes.

The intense convective vortices discussed above have also been observed in cold regions. Indeed, dust devils, waterspouts and tornadoes have been observed in subarctic regions (see Renno et al., 1998; Renno and Bluestein, 2001) and polar lows are similar to hurricanes (Emanuel and Rotunno, 1989). Moreover, large dust devils and intense cyclones (dust storms) are frequently observed on cold Mars. These observations are consistent with the proposed theory because it predicts that the intensity of convective vortices depends only on their thermodynamic efficiency and the enthalpy change between the environment and their core. Therefore, intense convective vortices can occur anywhere that these conditions are satisfied. In particular, they are likely to occur in regions of deep convective layer and large horizontal enthalpy gradient.

\subsection{Speculations about the causes of vortex walls and spiral bands}

Kangieser (1954), Sinclair (1969), Snow (1984) and Dowell et al. (2005), among others, suggest that a balance between inward pointing drag and outward pointing centrifugal forces concentrate dust and cloud particles in a ring around the centre of con- vective vortices such as dust devils and tornadoes. Snow (1984) points out that this explanation for the hollow nature of vortices requires the presence of sufficiently strong radial inflow. Indeed, Snow suggests that the balance between drag and centrifugal forces might explain hollow cores near the surface, where a strong radial flow is present, but that it probably does not explain what occurs above the surface layer. Rotunno (1977) show that strong radial flow is present only at the surface, and that the radial flow is weak and directed outward in the middle and upper portions of convective vortices. Moreover, Rotunno (1977) show in fig. 6.9 of his paper that a relative minimum in pressure develops at the region of maximum wind. He points out that above the surface, a ring of low pressure develops just outside the vortex wall. Rotunno (1977) argues that this ring of low pressure might explain the double walls observed in some tornadoes such as that studied by Golden (1973). The theory proposed in this paper provides a mechanism not only for the generation of radial flow towards the radius of maximum wind, but also for the generation of the relative pressure minimum discovered in this region by Rotunno (1977).

The wall cloud, a region of lowered cloud base nearly precipitation-free in the updraft of supercells, is considered to be a precursor to tornadogenesis (e.g. Golden and Purcell, 1978; Moller, 1978; Bluestein, 1980; Davies-Jones, 1986). Fankhausen (1976) and Bluestein et al. (1990) show evidence that the air under wall clouds is moister than the environment on the cases that they studied. Thus, the lifting of rain-cooled air might explain the formation of wall clouds. Davies-Jones (1986) suggests that wall clouds are manifestation of low-level mesocyclones. Wakimoto and Liu (1998) show that the wall cloud of a supercell studied during the VORTEX field experiment was centred on the storm's mesocyclone. However, they argued that the 2 $\mathrm{hPa}$ pressure drop in the wall cloud estimated from kinematic methods can account for only $20 \mathrm{~m}$ of the observed $1.4 \mathrm{~km}$ lowering in cloud base. The theory proposed in this paper suggests that pressure drops associated with increases in kinetic energy produce pressure drops much larger than that calculated by Wakimoto and Liu (1998). Indeed, the theory proposed in this paper can explain the lowering of the cloud base by a few hundred metres and the dynamical forcing of updrafts in the region. Thus, it suggests that both moistening by rain and dynamic effects contribute to the formation of wall clouds. This hypothesis can be tested with dynamic and thermodynamic measurements in wall clouds. The proposed theory is consistent with previous explanations for the hollow nature of convective vortices. Indeed, it provides a mechanism for flow convergence towards the vortex wall, in particular above the surface layer. The proposed theory predicts that the static pressure (as opposed to stagnation or total pressure) decreases with increases in kinetic energy. Thus, it predicts maximum pressure gradients in the regions of maximum wind. This might explain the hollow nature of intense convective vortices and the formation of rainbands in hurricanes. 
Small-scale convective vortices such as dust devils and waterspouts clearly show that dust and sea spray are lifted from the surface around the region of maximum tangential wind. Somewhere above the surface dust and sea spray frequently rise without much mixing, forming a hollow tube as illustrated in Fig. 2. We argue that this occurs not only because of peak shear stress in the region, but because non-hydrostatic pressure gradients are largest in the region of maximum tangential wind. These pressure gradients then dynamically force updrafts (by forcing mass convergence just above the surface) in the region of maximum tangential wind, and causes dust and sea spray to be rapidly transported upwards. In addition, the maximum pressure gradient at the radius of maximum wind combined with the centrifugal acceleration inhibits mixing and keeps dust, sea spray, and clouds droplets focused there. Moreover, the peak non-hydrostatic pressure drop somewhat above the surface pulls air not only from the vortex surroundings, but also from its core bringing clear and drier air from above to the lower portions of the vortex core and air from its centre at the surface layer upwards, creating its cone shaped base.

In moist convective vortices strong enough to produce pressure drops sufficient to cause condensation, the funnel cloud starts to form just below the cloud base where the air is nearly saturated. Equations (16a) and (23) predicts that in these regions, condensation starts first in the inflow region where the wind velocity is largest, as well as at the centre of the vortex. The prediction that large pressure drops are associated with increases in the flow's kinetic energy suggests that 'wall clouds' associated with strong tornadoes such as that illustrated in Fig. 3c are caused by pressure drop along the trajectory of air parcels spiraling towards the vortex. Thus, 'wall clouds' can also be forced by a dynamic pressure drop associated with jets of air spiraling towards the vortex. Equation (16a) suggests that a jet of $100 \mathrm{~m} \mathrm{~s}^{-1}$ causes a pressure drop of at least $50 \mathrm{hPa}$ and therefore could lower the cloud base by at least $500 \mathrm{~m}$. Moistening by precipitation must also contribute to the lowering of the base of wall clouds. These two ideas can be tested by thermodynamic measurements below them.

The mechanism described above might also explain the formation of thunderstorm walls around the eye of hurricanes, and perhaps even their spiral bands. Non-hydrostatic pressure gradients peak in regions of maximum wind and produces a dynamical forcing that aids the thermodynamical forcing of updrafts. Thus, eq. (16a) suggests that thunderstorms would be more prevalent along wind jets such as those found in hurricanes' spiral arms and rings of maximum wind.

\section{Conclusions}

The theory proposed in this paper offers a simple physical explanation for the most distinctive features of convective vortices and provides a thermodynamic foundation for understanding them on Earth and beyond. In particular, it predicts that the intensity of convective vortices depends on the depth of the convective layer via the thermodynamic efficiency, the enthalpy perturbation across them, and the existence of sources of vorticity (see Renno and Bluestein, 2001). It also predicts that non-hydrostatic pressure perturbations increase with the kinetic energy of air parcels spiraling towards the vortex. We speculate that this explains visible features of convective vortices such as wall clouds and the formation of funnel clouds. The proposed theory also predicts that the non-hydrostatic pressure drop at the region of maximum tangential wind is larger than the pressure drop at the surface.

Writing eq. (13) as

$$
\begin{gathered}
-(1-\gamma \eta) \frac{\Delta p}{\rho_{s}}+\gamma \eta\left[\left(c_{p} \Delta T+l_{v} \Delta q\right)+W_{\mathrm{irr}}^{a b}\right] \\
+\Delta \phi+\frac{\Delta v^{2}}{2}=0
\end{gathered}
$$

we can clearly see that the theory presented in this paper is a generalization of Bernouilli's equation to convective circulations. The kinetic and potential energy terms do not appear multiplied by the thermodynamic efficiency because these quantities have zero entropy. The negative sign in front of the first term is due to the fact that we define $\Delta p \equiv p_{a}-p_{b}$, but define changes in all other quantities as $\Delta\left({ }_{-}\right) \equiv\left({ }_{-}\right)_{b}-\left({ }_{-}\right)_{a}$.

\section{Acknowledgments}

The author would like to thank Prof. Richard Goody and Mr. Jasper Kok for reading the manuscript and making many useful suggestions, and Olivier Pauluis and two anonymous reviewers for making many constructive suggestions that improved the paper substantially. Finally, he would to thank the National Science Foundation for supporting this research under grant ATM 0402738 .

\section{References}

Adkins, C. J. 1968. Equilibrium Thermodynamics. Cambridge University Press, Cambridge, UK, 285 pp.

Adams, D. K. and Renno, N. O. 2005. Thermodynamic efficiencies of an idealized global climate model. Clim. Dyn. 25, 801-813, doi:10.1007/s00382-005-0071-y.

Barcilon, A. I. 1967. Vortex decay above a stationary boundary. J. Fluid Mech. 28, 65-84.

Bluestein, H. B. 1980. The University of Oklahoma severe storms intercept project-1979. Bull. Amer. Meteorol. Soc. 61, 560-567.

Bluestein, H. B., McCaul, E. W. and Byrd, G. P. 1990. Thermodynamic measurements under a wall cloud. Mon. Wea. Rev. 118, 794-799.

Bluestein, H. B., Weiss, C. C., French, M. M., Holthaus, E. M., Tanamachi, R. L. and co-authors. 2007. The structure of tornadoes near Attica, Kansas, on 12 May 2004: high-resolution, mobile, doppler radar observations. Mon. Wea. Rev. 135, 475-506.

Davies-Jones, R. P. 1986. Tornado dynamics. In: Thunderstorms: A Social and Technological DocumentaryVolume 2, 2nd Edition (ed.E Kessler). University of Oklahoma Press, Norman, OK, USA, 197-236. 
Dowell, D. C., Alexander, C. R., Wurman, J. M. and Wicker, L. J. 2005. Centrifuging of hydrometeors and debris in tornadoes: radarreflectivity patterns and wind-measurement errors. Mon. Wea. Rev. 133, 1501-1524.

Emanuel, K. A. 1986. An air-sea interaction theory for tropical cyclones. Part I: steady state maintenance. J. Atmos. Sci. 43, 585-604.

Emanuel, K. A. 1988. The maximum intensity of hurricanes. J. Atmos. Sci. 45, 1143-1155.

Emanuel, K. A. 1994. Atmospheric Convection. Oxford University Press, New York, 580 pp.

Emanuel, K. A. and Rotunno, R. 1989. Polar lows as arctic hurricanes. Tellus 41A, 1-17.

Emanuel, K. A. and Bister, M. 1996. Moist convective velocity and buoyancy scales. J. Atmos. Sci. 53, 3276-3285.

Fankhauser, J. C. 1976. Structure of an evolving hailstorm, Part II: thermodynamic structure and airflow in the near environment. Mon. Wea. Rev. 104, 576-587.

Ferri, F, Smith, P. H., Lemmon, M. and Renno, N. 2003. Dust devils as observed by Mars Pathfinder, J. Geophys. Res 108, 7/1-7/10, doi:10.1029/2000JE001421.

Golden, J. H. 1973. The Life Cycle of Florida Keys Waterspouts as the Result of Five Interacting Scales of Motion. PhD Dissertation. Florida State University, $371 \mathrm{pp}$.

Golden, J. H. 1974. The life cycle of Florida Keys' waterspouts. I. J. Appl. Meteorol. 13, 676-692.

Golden, J. H. and Purcel, D. 1978. Life cycle of the Union City, Oklahoma tornado and comparison with waterspouts. Mon. Wea. Rev. 106, 3-11.

Goody, R. 2000. Sources and sinks of climate entropy. Q.J.R. Meteorol. Soc. 126, 1953-1970.

Goody, R. 2003. On the mechanical efficiency of deep, tropical convection. J. Atmos. Sci. 60, 2827-2832.

Kangieser, P. C. 1954. A physical explanation for the hollow structure of waterspout tubes. Mon. Wea. Rev. 82, 147-152.

Koschmieder, E. L. 1993. Benard Cells and Taylor Vortices. Cambridge University Press, Cambridge, UK, 337 pp.

Landsea, C. W., Franklin, J. L., McAdie, C. J., Beven II J. L., Gross, J. M. and co-authors. 2004. A reanalysis of hurricane Andrew's intensity. Bull. Amer. Meteorol. Soc. 85, 1699-1712.

Lee, J. J., Samaras, T. M. and Young, C. R. 2004. Pressure measurements at the ground in an F-4 tornado. In: Proceedings of the $22^{\text {nd }}$ Conference on Severe Local Storms. Paper 11.4, Hyannis, MA.

Leibovich, S. 1978. The structure of vortex breakdown. Ann. Rev. Fluid Mech. 10, 221-246.

Leverson, V. H., Sinclair, P. C. and Golden, J. H. 1977. Waterspout wind, temperature and pressure structure deduced from aircraft measurements. Mon. Wea. Rev. 105, 725-733.

Lewellen W. S., Lewellen, D. C. and Sykes, R. I. 1997. Large-eddy simulation of a tornado's interaction with the surface. J. Atmos. Sci. 54, 581-605.

Lewellen, D. C., Lewellen, W. S. and Xia, J. 2000. The Influence of a Local Swirl Ratio on Tornado Intensification near the Surface. $J$. Atmos. Sci. 57, 527-544.

Liu, Y., Zhang, D.-L. and Yau, M. K. 1999. A multi-scale numerical study of hurricane Andrew (1992). Part II. Kinematics and Inner core structure. J. Atmos. Sci. 127, 2597-2616.

MacPherson, J. I. and Betts, A. K. 1997. Aircrafts encounters with strong coherent vortices over the boreal forest. J. Geophys. Res. 102, 2923129234.
Michaels, Timothy I. and Rafkin, S. C. R. 2004. Large eddy simulation of the convective boundary layer of Mars. Qt. J. R.l Meteorol. Soc. 130, 1251-1274.

Michaud, L. M. 1995. Heat to work conversion during upward convection: Carnot engine method. Atmos. Res. 39, 157-178.

Moller, A. R. 1978. The improved NWS storm spoters' training program at Ft. Worth, Tex. Bull. Amer. Meteorol. Soc. 59, 1574-1582.

Pauluis, O. and Held, I. M. 2002a. Entropy budget of an atmosphere in radiative-convective equilibrium. Part I: maximum work and frictional dissipation. J. Atmos. Sci. 59, 140-149.

Pauluis, O. and Held, I. M. 2002b. Entropy budget of an atmosphere in radiative-convective equilibrium. Part II: latent heat transport and moist processes. J. Atmos. Sci. 59, 125-139.

Pauluis O., Balaji, V. and Held, I. M. 2000. Frictional dissipation in a precipitating atmosphere. J. Atmos. Sci 57, 989-994.

Renno, N. O. 2001. Comments on "Frictional dissipation in a precipitating atmosphere". J. Atmos. Sci. 58, 1173-1177.

Renno, N. O. and Ingersoll, A. P. 1996. Natural convection as a heat engine: a theory for CAPE. J. Atmos. Sci. 53, 572-585.

Renno, N. O. and Bluestein, H. B. 2001. A simple theory for waterspouts. J. Atmos. Sci. 58, 927-932.

Renno, N. O., Burkett, M. L. and Larkin, M. P. 1998. A simple theory for dust devils. J. Atmos. Sci. 55, 3244-3252.

Renno, N. O., Nash, A. A., Lunine, J. and Murphy, J. 2000. Martian and terrestrial dust devils: test of a scaling theory using Pathfinder data. $J$. Geophys. Res. (Planets) 105 E1, 1859-1865.

Renno, N. O., Abreu, V., Koch, J., Smith, P. H., Hartogenisis, O. and co-authors. 2004. MATADOR 2002: a field experiment on convective plumes and dust devils, J. Geophys. Res. 109, E07001, doi:10.1029/2003JE002219.

Rotunno, R. 1977. Numerical simulation of a laboratory vortex. J. Atmos. Sci. 34, 1942-1956.

Samaras, T. M. 2004. An historical perspective of in-situ observations within tornado cores. In: Proceedings of the $22^{\text {nd }}$ Conference on Severe Local Storms. Paper 11.4, Hyannis, MA.

Sinclair, P. C. 1966. A Quantitative Analysis of the Dust Devil. $\mathrm{PhD}$ Dissertation. The University of Arizona, 292 pp.

Sinclair, P. C. 1969. General characteristics of dust devils. J. Appl. Meteorol. 8, 32-45.

Snow, J. T. 1984. On the formation of particle sheaths in columnar vortices. J. Atmos. Sci. 41, 2477-2491.

Sotiropoulos, F. and Ventikos, Y. 2001. The three dimensional structure of confined swirling flows with vortex breakdown. J. Fluid Mech. 426, $155-175$.

Souza, E. P., Renno, N. O. and Silva Dias, M. A. F. 2000. Convective circulations induced by deforestation. J. Atmos. Sci. 57, 2915-2922.

Spohn, A., Mory, M. and Hopfinger, E. J. 1993. Observations of vortex breakdown in a cylindrical container with a rotating bottom. Experiments in Fluids 14, 70-77.

Spohn, A., Mory, M. and Hopfinger, E. J. 1998. Experiments on vortex breakdown in a confined flow generated by a rotating disk. J. Fluid Mech. 370, 73-99.

Wakimoto, R. M. and Liu, C. 1998. The Garden City, Kansas, storm during VORTEX 95. Part II: the wall cloud and tornado. Mon. Wea. Rev. 126, 393-408.

Wurman, J. and Gill, S. 2000. Finescale Radar Observations of the Dimmitt, Texas (2 June 1995), Tornado. Mon. Wea. Rev. 128, 2135 2164. 